\title{
The synergy between Expanded Program on Immunization and Polio Eradication Initiative in Pakistan: a policy and program exploration
}

\author{
Zaeem Haq ${ }^{1}$, Arshad K. Chandio' ${ }^{2}$ Shamsa Zafar ${ }^{3}$, Faisal Iqbal', Ayesha Naeem ${ }^{5}$, Saad Karim ${ }^{5}$ \\ 1 Health Services Academy, Islamabad, Pakistan, ${ }^{2}$ Medicine, KRL Hospital, Islamabad, Pakistan, ${ }^{3}$ Gynaecology \& Obstetrics, PAF Hospital, Islamabad, \\ Pakistan, ${ }^{4}$ International Labour Organization, Islamabad, Pakistan, ${ }^{5}$ Child Advocacy International, Islamabad, Pakistan \\ Keywords: polio eradication, routine immunization, expanded program on immunization
}

https://doi.org/10.29392/001c.26146

Journal of Global Health Reports

Vol. 5, 2021

\section{Background}

Until recently, Pakistan had achieved significant milestones in polio eradication, and was close to becoming polio-free. The country has an Expanded Program on Immunization (EPI) since 1978 and a Polio Eradication Initiative (PEI) since 1994. PEI and EPI need to carry out synergized activities during the last leg of polio eradication; EPI taking over the responsibility for a polio-free Pakistan. Knowing the understanding and implementation of the synergy between the two programs is important at this critical juncture.

\section{Methods}

We carried out a national-level exploration, comprised of 30 individual interviews and eight focus groups. The objectives were: (i) to explore the current understanding of the PEI-EPI synergy and its operationalization, especially for the zero-dose children at various levels, and (ii) to explore the context of the two programs, the synergy-related enablers and barriers, and suggestions to enhance synergy in the future. The participants included officials from various tiers along with health workers and vaccinators.

\section{Results}

Four themes emerged. First, "synergy” has different meanings for different stakeholders; hence variable implementation models. Second, some synergies (e.g., sharing of surveillance data by PEI) are in place while additional (e.g., transferring human resources and enhancing community engagement) can be created. Third, the EPI and the PEI have become two unequal programs because of political factors, visibility, and funding. Fourth, the situation can be helped through clear policy and operational guidelines including roles and responsibilities.

\section{Conclusions}

All stakeholders understand the importance of synergy. The willingness for the adoption of the interventions instituted by the PEI exists on both sides. The EPI, however, needs to enhance its capacity to absorb these interventions and improve service delivery. Given the context in which one partner has gained more political ownership, funding, and visibility than the other, the realization of the international donors, and the guidance from the Ministry of National Health Services, Regulations, and Coordination are essential for ensuring the polio legacy and achieving and maintaining the status of a polio-free Pakistan.

The world is welcoming the effectiveness of the COVID-19 vaccine, including the reduction in severe acute respiratory syndrome coronavirus 2 (SARS-CoV-2) related morbidity and mortality. ${ }^{1,2}$ Ensuring routine immunization while dealing with the pandemic has also been a priority discussion. ${ }^{3}$ At the same time, past and present studies from low to middle-income countries have documented the systemic challenges that their immunization programs face because of which vaccine delivery has been and will be dif- ficult. ${ }^{4,5}$ Because of these challenges, vaccine-preventable diseases like poliomyelitis still exist despite the availability of vaccines and their delivery systems.

After Africa being declared polio-free, only two South Asian countries are reporting cases of poliomyelitis. ${ }^{6}$ One of them is Pakistan, which started its Polio Eradication Initiative (PEI) in 1994 and went on to carry out polio campaigns over the next two decades. A characteristic factor is Pakistan's isolated subpopulations with persistent polio 
that benefit little from high immunity among the surrounding population and continue behaving as a reservoir of the wild polio virus (WPV1). ${ }^{7}$ Moreover, places in Pakistan continue to have inadequate sewerage systems and have grappled with terrorism for a long time. ${ }^{8}$ The former facilitates the survival of the poliovirus in the environment while the latter often turns a health problem into a security issue. ${ }^{9}$

With 306 WPV1 cases- the world's highest in 2014- Pakistan declared polio an emergency and established an Emergency Operations Centre (EOC), directly supervised by the Prime Minister at the federal and by the Chief Ministers at the provincial level. ${ }^{10}$ The EOC develops a National Emergency Action Plan (NEAP) each year that sets the strategic directions. ${ }^{10}$ Because of EOC's focus on the implementation, the WPV1 cases consistently declined till 2017, with only 8 cases in that year. A surge, however, was observed in 2018 with 12 cases, followed by 147 cases in 2019. At the same time, Pakistan also had an outbreak of circulating Vaccine Derived Polio Virus2 (cVDPV2) with 22 reported cases in 2019. Owing to COVID-19 in the year 2020, the EOC paused polio campaigns from March till August. Upon resumption, the cases declined significantly, with 84 WPV1 cases in 2020 and only one in 2021. Similarly, the 135 cVDPV2 cases during 2020 decreased to seven in 2021 till this report. ${ }^{11}$

Pakistan's Expanded Program on Immunization (EPI), which currently provides routine immunization (RI) against 11 diseases, including polio, was established in 1978. The most recent EPI figures ${ }^{12}$ show that about $70 \%$ of children receive complete vaccination, which means that about $1 /$ $3^{\text {rd }}$ of Pakistani children are still not fully immunized. It is this group of uncovered children, which, if not immunized, can lead to an addition in polio cases and undermine the success. Also significant are the regional disparities; within the overall national figures of $70 \%$ is Balochistan, where only $27 \%$ of children could receive full immunization. ${ }^{12}$ The Quetta and Qila Abdullah block from this province form one of the three polio reservoir hubs, also called Super HighRisk Union Councils (SHRUCs). ${ }^{13}$

In countries like Pakistan, where PEI and EPI exist as two independent programs, a complex interplay of relationships, processes, and outcomes is observed. With the ultimate common goal of preventing children from disease, the two programs should work in synergy and complement each other. However, their target population clusters with polio cases have a large number of unimmunized or under-immunized children, leading to a high risk of continued circulation and outbreaks. ${ }^{14}$ According to the NEAP, out of the 147 WPV1 cases reported in 2019 from Pakistan, 93 (63\%) were zero-dose, i.e., they had received no vaccination; proof of the weak RI in the country. ${ }^{13}$

Several studies have shown that both programs can support each other in achieving targets. ${ }^{15-17}$ However, an important argument is that these complementary effects are not automatic; they have to be deliberately planned. ${ }^{18}$ Guidance is available for this planning to absorb polio resources into immunization and other public health programs during post-polio times. ${ }^{19,20}$ The Global Polio Eradication Initiative's Polio Eradication and Endgame Strategic Plans 2013-2018 and 2018-2023 outline a roadmap for successfully eradicating polio. ${ }^{14,21}$ The plans include replacing the oral polio vaccine (OPV) with the injectable counterpart called IPV, an enhanced system of RI, and effective planning for the 'legacy' of polio resources to be mainstreamed into other public health programs. ${ }^{21,22}$

According to the WHO, 'legacy' pertains to the post-polio phase and consists of three elements: mainstreaming polio, leveraging knowledge, and transitioning assets and resources into the broader system. ${ }^{21}$ The Government advised synergy between EPI and PEI to ensure the legacy of polio and ultimately the sustainability of the polio-free status of the country. ${ }^{10}$ How EPI and PEI in Pakistan work in a "synergized" fashion to pave the way for legacy is an important question. Minimum information, however, is available about it. Our study bridges this gap by exploring the concept of synergy and its implementation and barriers and enablers to this essential prerequisite of polio legacy.

\section{METHODS}

\section{CONCEPTUAL FRAMEWORK}

Handler and colleagues proposed a framework that explains meaningful relationships among various health system components, providing a basis to examine their performance. According to them, the macro-context and the broader health system interact with the system's structural capacities to influence the implementation and the outcomes. ${ }^{23}$ We adapted this framework (see Figure 1) for our study proposing that within the macro-context and system guidance, the synergistic interactions can take place between structural units (EPI and the PEI), leading to activities required for achieving the poliovirus interruption.

Specific objectives of the study included:

1. To explore the current understanding of the PEI-EPI synergy and its operationalization, especially for the zero-dose children at various levels.

2. To explore the context of the two programs, the synergy-related enablers and barriers, and suggestions to enhance synergy in the future.

\section{STUDY DESIGN, PARTICIPANTS, AND SETTING}

Using a narrative design, ${ }^{24}$ this national study covered all of Pakistan. The participants included officials from the Ministry of National Health Services, Regulations and Coordination (NHSR\&C) and all four provinces and representatives from civil society and partner organizations working for immunization. The EPI vaccinators and communitybased vaccination (CBV) workers of PEI in two provinces, i.e., Punjab and Khyber Pakhtunkhwa (KP), were also included. Lack of time and resources was a limiting factor that led us to restrict the district-level exploration in two provinces that represented the high and low end of the success of polio immunization. All officials of government and partner agencies working at the federal, provincial, and district levels having an interface with immunization activities were invited. We interviewed the participants in person at their offices, health facilities, and some neutral places (e.g., cafés). The purpose was to have these discussions where the respondents were easily accessible and comfortable while talking about the issue under discussion. A small number of 


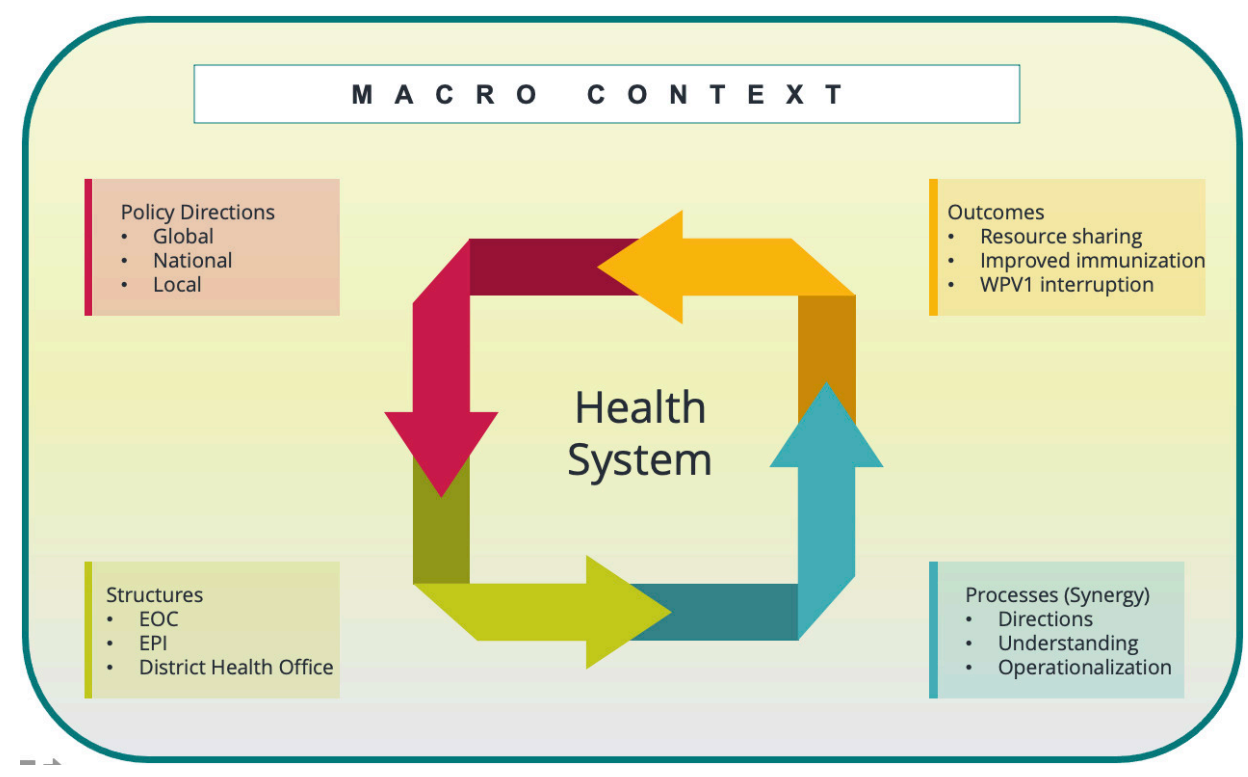

Figure 1. Conceptual framework to explore stakeholders' viewpoint for synergized immunization outcomes in Pakistan

participants were also interviewed over the telephone.

\section{DATA COLLECTION, PROCESSING, AND ANALYSIS}

We developed the interview/discussion guide using the focus areas outlined in the conceptual framework of this study (Figure 1). We directed our inquiry at exploring the current understanding of EPI-PEI synergy and its operationalization at various levels. We also discussed the context in which two programs implemented synergy and the perceived enablers and barriers, along with suggestions for improving synergy and ensuring legacy in the future. A team of two experienced interviewers ( $\mathrm{ZH}$ and $\mathrm{SZ}$ ) conducted the semi-structured interviews and Focus Group Discussion (FGDs).

Individual, semi-structured interviews were deemed appropriate for program managers and policymakers to fit their busy schedules, while FGDs were adopted for vaccinators and CBV workers, as it enabled collecting information from more people in a short time. Written consent was obtained before beginning the discussions. At the start of an FDG, the moderator briefly described the objectives and asked for tape-recording the discussion. Detailed, verbatim notes of the discussion were taken where permission for tape-recording was not granted. A typical interview started with broad questions about the concept of synergy and its implementation. The discussion then evolved depending upon the responses and the program area in which the respondent had had a specific role.

We conducted most of these interviews and discussions using a mix of Urdu and English languages, and their duration ranged from 30 minutes to about two hours. No incentive or payment was offered to the respondents at any point. Data collection was carried out from July 15, 2017 to November 7, 2017. The study was supported through funds provided by Gavi, the Vaccine Alliance, in partnership with Health Services Academy Islamabad and UNICEF. Ethical clearance was obtained from the Institutional Review Board (IRB) of the Health Services Academy Islamabad. The study was conducted as part of an embedded implementation research $^{25}$ and required the officials' involvement from the participant programs to ensure the translation of study findings into the program's policy and practice. However, to avoid the social desirability bias during data collection, these officials were not included in the study's data collection or analysis part.

Thematic content analysis ${ }^{26}$ was carried out on full transcripts of the interviews and FGDs by following the recommended steps. ${ }^{27}$ Two members of the study team carried out the analysis. First, they agreed on a method of coding guided by the conceptual framework. Following this, they independently analyzed three initial transcripts and met again to see the concordance between their coding. The codes that did not match were discussed to reach a consensus. The process helped in the development of a final code sheet which was used to identify the significant statements across individual interviews. ${ }^{26}$ Subsequent readings of these significant statements helped in identifying subthemes emerging within these patterns. We gave equal attention to the divergent themes- points not shared by most respondents but deemed significant. ${ }^{26,28}$

\section{RESULTS}

Overall, a total of 112 men and women participated in the study. Among them, 30 were individually interviewed, while 82 participated in the eight FGDs at the district level (see Table 1). The themes and sub-themes that emerged from these interviews and discussions are summarized below.

\section{CLARITY ABOUT SYNERGY}

Senior officials from the NHSR\&C appeared confident about the idea of synergy and its implementation. According to 
Table 1. Summary of participants to interviews and FGDs $(\mathbf{n}=112)$

\begin{tabular}{|c|c|c|c|c|c|}
\hline \multicolumn{6}{|c|}{$\begin{array}{l}\text { Individual interviews } \\
\qquad(n=30)\end{array}$} \\
\hline \multirow[t]{2}{*}{ Level } & \multicolumn{4}{|c|}{ Category: Ministry/department, programme or organization } & \multirow[t]{2}{*}{ Total } \\
\hline & $\begin{array}{l}\text { Ministry/Department of } \\
\text { Health }\end{array}$ & EPI & $\begin{array}{c}\text { Polio } \\
\text { Programme }\end{array}$ & $\begin{array}{c}\text { Partner } \\
\text { organization }\end{array}$ & \\
\hline Federal & 1 & 2 & 3 & 4 & 10 \\
\hline Provincial & 2 & 5 & 4 & 4 & 15 \\
\hline District & 1 & 2 & 2 & 0 & 5 \\
\hline Sub-total & 4 & 9 & 9 & 8 & 30 \\
\hline \multicolumn{6}{|c|}{$\begin{array}{l}\text { Focus group discussions (FDGs) } \\
\qquad(\mathrm{n}=82)\end{array}$} \\
\hline Province & District & Category & $\begin{array}{l}\text { Number of } \\
\text { FGDs }\end{array}$ & \multicolumn{2}{|c|}{ Number of Participants } \\
\hline Punjab & Rawalpindi & Vaccinator & 4 & \multicolumn{2}{|l|}{28} \\
\hline $\begin{array}{l}\text { Khyber } \\
\text { Pakhtunkhwa }\end{array}$ & Peshawar & CBV worker & 3 & \multicolumn{2}{|l|}{46} \\
\hline $\begin{array}{l}\text { Khyber } \\
\text { Pakhtunkhwa }\end{array}$ & Peshawar & $\begin{array}{c}\text { CBV } \\
\text { Supervisor }\end{array}$ & 1 & \multicolumn{2}{|l|}{8} \\
\hline Sub-total & & & 8 & \multicolumn{2}{|l|}{82} \\
\hline Total participar & al interviews and FGDs) & & & \multicolumn{2}{|c|}{112} \\
\hline
\end{tabular}

EPI: Expanded program on Immunization CBV: Community based vaccination

these officials, essential steps have been taken, and clarity is provided in the NEAP document. The objective is to make optimal use of polio resources and improve RI in the country.

"The inclusion of IPV into the EPI schedule is in place since 2015. NEAP, which is a policy document, emphasizes the synergy between the two. The agenda of the prime minister's task force meetings already contains strengthening of RI all the time. A synergy team has been constituted. All of this would improve RI, which is necessary for maintaining the country's polio-free status, once polio program ceases to exist." -NHSRC official, Islamabad.

While identifying NEAP as the key policy document, the participants from the provincial- and district levels noted that the district and below levels do not get a chance to provide input. Moreover, the operational guidelines agreed by all parties are not available, leading to confusion.

\footnotetext{
"The root of synergy lies in NEAP. It identifies that polio cases are not being controlled due to weak RI. So, one of the key strategies to eradicate polio is to boost RI that will ultimately help polio eradication. There is, however, no framework to guide all stakeholders on sharing the data and the micro-plans, etc." -EOC official, Balochistan.
}

When asked, field staff from the districts were ignorant of the concept of synergy and could only mention the challenges they have about their work when it comes to working with their immunization counterparts (EPI and PEI) in the district. The EPI staff mainly complained about their extensive work, including the support they provide to polio SIAs, while PEI staff was more critical of the EPI program.
"We face many refusals because of the EPI program. We put effort into convincing the community and send them to a health center, but the staff there say there is no vaccine available, and they also misbehave. As a result, these people not only refuse routine injections, they also refuse $O P V$ next time." CBVs, KP.

\section{OPERATIONALIZATION OF SYNERGY}

In the absence of operational guidelines, the participants listed activities of varied nature that they thought was a mark of synergy between the two programs. However, a recurring theme was the data collection by polio staff about the RI, which they were doing initially in priority districts from the province of Sindh, KP, and Balochistan. The focus of synergy, according to these respondents, was the CBV workers and their data collection.

\footnotetext{
"Recently, the EOC instructed all CBV workers to conduct a micro-census for EPI. When our teams visit house to house, they collect data on the newborns that have not been vaccinated yet. They share this zero RI data with the EPI of that locality, and the exercise will continue after every six months."- PEI official, Islamabad.
}

The federal and provincial managers and other senior officials from EPI also mentioned the micro-census that PEI field staff has started and will help in enhancing the RI. However, EPI's district and community-level staff were not agreeable to the definition of zero-dose children and the relevant data being shared.

"CBVs go from door to door to collect data about all vaccinations. But they count all children who have not received any vaccination as zero-dose. In reality, EPI vaccinators 
cannot vaccinate all babies, especially those born at their homes, immediately after birth. We call a baby zero-dose if it is 40 days old and still not vaccinated." - EPI vaccinators \& officials.

The other examples for synergy were the procurement and logistics of polio vaccine provided by the EPI, the experiment of Punjab province giving program leadership of both PEI and EPI to the same individual, using the same trainers by both programs in the Sindh province, and inviting the counterparts to a program's review meetings in the Sindh province.

\begin{abstract}
"In Sindh, EPI is sitting on the review meetings of EOC. It is a good opportunity for them to look at the program indicators and how EOC traces them to learn lessons. Currently, there are 27 indicators and targets used for PEI that can be transferred to and adopted by EPI." - EOC official, Sindh.
\end{abstract}

\section{ENABLERS AND BARRIERS}

The high-level commitment from the Ministry of NHSRC and the leadership of both PEI and EPI programs is the most significant enabler. There seems to be a realization that it will be tough for PEI to eradicate polio from Pakistan all by itself. Likewise, the RI activities can receive massive support from the extensive field force that PEI has put together over the years.

\section{"The Ministry is fully committed to the idea of synergy be- cause there is no other way out of the complex polio situ- ation in communities where people suspect the polio-only campaigns and vociferously demand nutrition, maternal and child health, and WASH services. This operational re- search on synergy is another proof of our seriousness." NHSRC official}

The successful introduction of IPV to the immunization schedule is another enabling synergistic activity for moving towards polio eradication. Introducing IPV is a critical element of the endgame plan and global readiness to manage risks associated with OPV type 2 withdrawal, which Pakistan did in 2015 before introducing the injectable vaccine.

\begin{abstract}
"The endgame plan advises introducing IPV in all OPVonly using countries by the end of 2015. The primary role of IPV, which EPI vaccinators are giving, will be to maintain immunity against type 2 poliovirus while removing OPV type 2 from the schedule." EPI official, Islamabad.
\end{abstract}

Some barriers to synergy were also mentioned in the discussions. The participants highlighted that although both programs are working on immunization, many factors make them different. Everything, therefore, cannot be synergized, and one has to prioritize where to begin the process.

\begin{abstract}
"To begin with, these are two unequal programs in many respects. One is highly sophisticated, and the other is very basic. One enjoys global, national, and local political ownership and adequate funding, while the other is just beginning to get attention. Then the target children, their ages and context are different."-EPI official, Islamabad.
\end{abstract}

Another impeding factor is that while EPI's vaccinator also performs for PEI, the polio workers do not have any ac- tivities that support RI during the polio campaigns, except for collecting data of un-immunized children. In addition, vaccinators also face a lack of supplies for RI, which is not the case with polio immunization.

"We do not find enough time to fulfill our regular duties
and tasks due to NIDs [National Immunization Days] and
repeated polio activities, and it is affecting our routine
work - we cannot cover our children in routine. Besides,
we do have vaccines, but syringes, immunization cards,
and stationery are usually deficient." - Vaccinators,
Rawalpindi.

\section{SUGGESTIONS FOR THE FUTURE}

Bringing on some level of conceptual clarity and providing implementation guidelines was mentioned by many participants as an action point. They repeatedly highlighted the varied understanding of synergy within and between the two programs and resultant confusion and implementation gaps. They also shared their opinion about ways that can help address this confusion and resultant gaps.
"Although we are talking about synergy, we have not yet designed any framework on how we are going to do it. For example, if we are engaging $C B V$ s for RI, how would that happen? And then what would happen when polio cam- paigns end and $C B V$ support is withdrawn. There is no as- surance that $C B V$ s will be available then. Clearly, we need policy directions and methods to implement them." - Of- ficial, a Partner organization.

Participants from various levels, especially the field level, repeatedly mentioned the importance of addressing the problem of zero-dose children to improve the PEI-EPI synergy. For the PEI, the high number of zero-dose children was a significant obstacle in eradicating polio. On the other hand, EPI viewed the zero-dose data as an effort being used to justify PEI's failure to eradicate polio from the country.
"The polio worker should collect the zero-dose data and share it with EPI promptly. While collecting this data, the polio worker should also inform the family about the EPI center and the outreach schedule of the area vaccinator so that that family can reach this center or the vaccina- tor. The EPI worker should use the polio worker's lists and share the list of immunized children back with PEI. All of this needs to happen under an agreed definition and strat- egy.” EPI official, Punjab.

Several EPI and PEI participants, especially from among the field workers, mentioned that the EPI needs to enhance its capacity and strengthen systems. They mentioned that adequate vaccinators, the ongoing training and promotion of vaccination staff, and sufficient supplies are required for better outcomes.

"While macro issues, like procurement, logistics of vaccines and storage, etc., have been revamped majorly, the infrastructure at the grass-root level needs focus. For example, we need to ensure that vaccinators are available everywhere, health facilities are all well-staffed and functional, and efficient logistics available for vaccinating every child." - EPI official, Islamabad. 


\section{DISCUSSION}

This study explored the understanding and implementation of synergized activities between the EPI and the PEI in Pakistan, a strategy that the country decided to adopt towards the tail end of polio eradication. A recurrent theme among the participants was the deficient clarity about the concept of synergy and its operationalization. As a result, there is a lack of acknowledgment of the interdependence on both sides of the program. Despite the achievements like the introduction of IPV, there is a confusing implementation and impeded future thinking about reaching a polio-free Pakistan and sustaining that status through a system that leverages the knowledge and resources generated by both the programs. The EPI-PEI combo also needs synchronizing with other health programs (e.g., maternal and child health) and the broader health system, requiring a clear, sequential, and strategic approach towards integration.

The issue of zero-dose children and the administration of birth dose is the major obstacle faced by the field staff. The global guidance like the Polio Endgame Strategy 2018-23 and the Gavi 5.0 strategy emphasize the strengthening of RI and transition of resources to EPI. ${ }^{14,29}$ The NEAP mentions synergy and ISD but does not crystallize the thinking to bring it down to the core issues of zerodose, birth dose, and integrated demand generation. ${ }^{13}$ The EPI guidance also falls short on these aspects. In accordance with a definition agreed by both the programs, the leadership needs to ensure that actionable information on zerodose children reaches the field force on either side of the immunization aisle. When polio workers collect data from the family is the best opportunity to assist the EPI in demand generation activities. Polio teams can achieve this if they inform the family about the immunization need of their child, the place where the child can get the vaccine, and the time when it is the easiest for the family.

In addition to less clarity about the concept, and insufficient knowledge of the operational guidelines, the participants from district and field-level seemed disconnected from the entire process. While they highlighted issues like zero-dose and birth-dose, which are the fundamental problems underlying a lack of synergy, it appeared that they had not been consulted during the formulation of this vital pillar of immunization programming in Pakistan. Past studies $^{27}$ on policymaking mention the problems of top-down, ad-hoc policies developed without any need-assessment or trickle-up of the input, which is visible in the case of PEIEPI synergy as well.

Interestingly, some top-down elements were also found missing, which the global guidance recommends. ${ }^{14}$ For example, we did not hear from participants about leveraging knowledge and transferring resources. None of the discussants brought up the issue of building upon the knowledge that PEI has gathered over decades, in terms of surveillance in difficult-to-reach areas, reaching the last child to provide OPV, understanding, and addressing people's perceptions about the vaccine, including its acceptance and hesitancy. We did not hear about the steps under consideration when CBV workers will be withdrawn, and household information about zero-dose children will still be required. According to the WHO, 50 percent of polio staff should spend time on RI activities to move towards legacy. In other countries, polio staff spends about 47 percent of their time on RI activities, while the staff in Pakistan spends only 18 percent of their time on joint activities. ${ }^{30}$ Nevertheless, in our discussions, we did not hear about this transitioning of assets and resources.

Underneath the appreciation of synergy expressed by higher officials, the perception of two unequal programs whose district and community-level staff do not have confidence and mutual trust was palpable. The feeling that PEI enjoys a higher level of ownership from the national Government and international donors, and better access to the highest level of governance in the country, impedes the input from EPI and needs attention. The recent significant contributions of both programs to the country's response to COVID-19 underscores that both can perform and contribute to public health delivery when they are given a level playing field. At the National Command and Operations Centre, ${ }^{31}$ where both worked under a single leadership, PEI contributed via its country-wide surveillance of COVID-19 cases and EPI through its vaccine delivery across the country.

Some limitations of this study must also be mentioned. First, examining the development of synergy as a policy or strategy was not within the scope of our research. During the study, however, we found that the essentials of policy development are lacking, but we could not dwell on this aspect as that would divert from the main study objectives. Second, due to the lack of time and resources, we did not include community perspectives; whether they view PEI and EPI as a singular program or two different entities could have brought valuable insights from the end user's perspective. This perspective may influence vaccine acceptance and hesitancy behaviours. Third and last, our task was to examine the synergy between PEI and EPI. However, the concept of synergy and integration transcends immunization and embraces other health programs like Maternal and Child Health $(\mathrm{MCH})$ care, primary healthcare through Lady Health Worker (LHW), and other functions of the health sector. The role of these sectors in helping improve immunization is important and must be examined in detail.

\section{CONCLUSIONS}

The immunization stakeholders in Pakistan understand the importance of synergy between PEI and EPI, and there is a willingness on both sides to support each other. Both programs have demonstrated their strengths and contributed through their respective role to Pakistan's response to COVID-19. The same level of coordinated response can be made possible for polio eradication, provided the concept and implementation of synergy between the two are clarified, and a future course of action is defined for both the programs. Integration with other programs and the broader health sector is also essential, but pragmatism requires that the two programs, which have immunization as a common goal, firm up their synergism before embarking on integration with other sectors. 


\section{ACKNOWLEDGMENTS}

We are extremely grateful to all the participants belonging to federal, provincial and district programs including the vaccinators and CBVs for their valuable time and participation.

\section{FUNDING}

This study is supported by Global Alliance for Vaccination and Immunization (GAVI) through a grant jointly administered by UNICEF and Health Services Academy, Islamabad, Pakistan.

\section{AUTHORSHIP CONTRIBUTIONS}

ZH and SZ conceptualized the study and developed tools, $\mathrm{ZH}$ along with SZ, FI, AN and SK collected and analysed the data, and developed the first draft. All authors contributed to successive drafts till the final submission.

\section{COMPETING INTERESTS}

The authors completed the Unified Competing Interest form at http://www.icmje.org/disclosure-of-interest/ (available upon request from the corresponding author), and declare no conflicts of interest.

\section{CORRESPONDENCE TO:}

Zaeem ul Haq, MBBS, MCPS, PhD.

Health Services Academy, Chak Shahzad, Islamabad, Pakistan.

drzaeem@hotmail.com

Submitted: July 18, 2021 GMT, Accepted: July 25, 2021 GMT 


\section{REFERENCES}

1. Leshem E, Wilder-Smith A. COVID-19 vaccine impact in Israel and a way out of the pandemic. The Lancet. 2021;397(10287):1783-1785. doi:10.1016/s014 $\underline{0-6736(21) 01018-7}$

2. Wise J. Covid-19: Is vaccination roll out reducing cases and deaths in the UK? BMJ. 2021;372(February):n506. doi:10.1136/bmj.n506

3. Wong YJ, Lee SWH. COVID-19: A call for awareness or mandatory vaccination even in pandemics? J Glob Health. 2021;11:1-3. doi:10.7189/jogh.11.03005

4. Oyadiran OT, Usman SA, Osoba ME, Olukorode SO, Lucero-Prisno III DE. Towards effective and efficient COVID-19 vaccination in Nigeria. Journal of Global Health Reports. 2021;5:1-6. doi:10.29392/001c.21404

5. Haq Z, Shaikh BT, Tran N, Hafeez A, Ghaffar A. System within systems: Challenges and opportunities for the expanded programme on immunisation in Pakistan. Health Res Policy Sys. 2019;17(1). doi:10.118 6/s12961-019-0452-z

6. Global Polio Eradication Initiative. History of polio. Published 2018. Accessed October 5, 2020. http://poli oeradication.org/polio-today/history-of-polio/

7. Thompson KM, Tebbens RJD. Lessons From the Polio Endgame: Overcoming the Failure to Vaccinate and the Role of Subpopulations in Maintaining Transmission. The Journal of Infectious Diseases. 2017;216(suppl_1):S176-S182. doi:10.1093/infdis/iix1 $\underline{08}$

8. Hussain SF, Boyle P, Patel P, Sullivan R. Eradicating polio in Pakistan: an analysis of the challenges and solutions to this security and health issue. Global Health. 2016;12(1):1-9. doi:10.1186/s12992-016-019 $\underline{5-3}$

9. Riaz H, Rehman A. Polio vaccination workers gunned down in Pakistan. The Lancet Infectious Diseases. 2013;13(2):120. doi:10.1016/s1473-3099(1 2)70344-4

10. Government of Pakistan, Ministry of NHSR \&C. National Emergency Action Plan for Polio Eradication 2015-2016.

11. Global Polio Eradication Initiative-Pakistan. Pakistan Status. Published 2020. Accessed May 28, 2021. http://polioeradication.org/where-we-work/pak istan/
12. Pakistan Bureau of Statistics. Pakistan Social and Living Standards Measurement Survey.; 2020. https://w ww.pbs.gov.pk/sites/default/files//pslm/publications/ pslm_district 2019-20/Key_Finding_Report_of_PSL M_District_Level_Survey_2019-20.pdf

13. Government of Pakistan, Ministry of NHSR\&C. National Emergency Action Plan for Polio Eradication -2020.; 2020.

14. World Health Organization. Polio Endgame Strategy 2019-2023: Eradication, Integration, Certification and Containment. Vol 19.; 2019. http://pol ioeradication.org/wp-content/uploads/2019/06/englis h-polio-endgame-strategy.pdf

15. Closser S, Cox K, Parris TM, et al. The Impact of Polio Eradication on Routine Immunization and Primary Health Care: A Mixed-Methods Study. The Journal of Infectious Diseases. 2014;210(Suppl 1):S504-S513. doi:10.1093/infdis/jit232

16. Hopkins DR. Disease Eradication. N Engl J Med. 2013;368(1):54-63. doi:10.1056/nejmra1200391

17. Michael CA, Waziri N, Gunnala R, et al. Polio Legacy in Action: Using the Polio Eradication Infrastructure for Measles Elimination in Nigeria-The National Stop Transmission of Polio Program. The Journal of Infectious Diseases. 2017;216(suppl_1):S373-S379. doi:10.1093/infdis/jix0 $\underline{14}$

18. Loevinsohn B, Aylward B, Steinglass R, Ogden E, Goodman T, Melgaard B. Impact of Targeted Programs on Health Systems: A Case Study of the Polio Eradication Initiative. Am J Public Health. 2002;92(1):19-23. doi:10.2105/ajph.92.1.19

19. van den Ent MMVX, Mallya A, Sandhu H, et al. Experiences and Lessons From Polio Eradication Applied to Immunization in 10 Focus Countries of the Polio Endgame Strategic Plan. The Journal of Infectious Diseases. 2017;216(Suppl 1):S250-S259. do i:10.1093/infdis/iix047

20. Rutter PD, Hinman AR, Hegg L, et al. Transition Planning For After Polio Eradication. The Journal of Infectious Diseases. 2017;216(Suppl 1):S287-S292. do $\mathrm{i}: 10.1093 /$ infdis/jix026

21. World Health Organization. Polio Eradication \& Endgame Strategic Plan 2013-2018.; 2013. 
22. Abdelwahab J, Dietz V, Eggers R, et al. Strengthening the Partnership Between Routine Immunization and the Global Polio Eradication Initiative to Achieve Eradication and Assure Sustainability. Journal of Infectious Diseases. 2014;210(suppl 1):S498-S503. doi:10.1093/infdis/jiu0 $\underline{41}$

23. Handler A, Issel M, Turnock B. A Conceptual Framework to Measure Performance of the Public Health System. Am J Public Health. 2001;91(8):1235-1239. doi:10.2105/ajph.91.8.1235

24. Cresswell JW. Qualitative Inquiry and Research Design: Choosing Among Five Approaches. 3rd ed.; 2013.

25. Shahabuddin A, Sharkey A, Khalid F, et al. An embedded implementation research initiative to tackle service delivery bottlenecks in the expanded programme on immunisation in Pakistan: Overview and reflections. J Glob Health. 2021;11. doi:10.7189/jo gh.11.06003

26. Patton MQ. Qualitative Research \& Evaluation Methods. 3rd ed.; 2002.
27. Haq Z, Hafeez A, Zafar S, Ghaffar A. Dynamics of evidence-informed health policy making in Pakistan. Health Policy and Planning. 2017;32(10):1449-1456. $\underline{\mathrm{d}}$ oi:10.1093/heapol/czx128

28. Miles M, Huberman M. Qualitative Data Analysis: An Expanded Sourcebook. 2nd ed.; 1994.

29. Gavi, The Vaccine Alliance. Gavi 5. 0 The Alliance's 2021-2025 Strategy.; 2019. https://www.gav i.org/sites/default/files/board/minutes/2019/06 - Gavi 5.0 The Alliances 2021-2025 Strategy.pdf

30. van den Ent MMVX, Swift RD, Anaokar S, Hegg LA, Eggers R, Cochi SL. Contribution of Global Polio Eradication Initiative-Funded Personnel to the Strengthening of Routine Immunization Programs in the 10 Focus Countries of the Polio Eradication and Endgame Strategic Plan. The Journal of Infectious Diseases. 2017;216(Suppl 1):S244-S249. doi:10.1093/i nfdis/jiw567

31. Government of Pakistan. National Command and Operation Center. Situation Reports. Published 2021. Accessed May 25, 2021. https://ncoc.gov.pk/ 